\title{
The potential of trefoil proteins as biomarkers in
} \section{human cancer}

"The question is not only do the malignant cells express the proteins but also to where are the proteins being secreted? ... if trefoil proteins are being secreted by normal or quasi-normal epithelial cells into ductal lumina, this is indicative of good differentiation and prognosis but if they are being secreted by invasive malignant cells into the stroma then this is indicative of a metastatic phenotype..."

KEYWORDS: adenocarcinoma $\approx$ androgen receptor $\approx$ estrogen receptor $₫ H E R 2$ - predictive $\mid$ prognostic $\|$ stratified medicine $\mid$ TFF1 $\mid$ TFF2 - TFF3 - trefoil factor

Knowledge about the molecular etiology of human cancer underwent a seismic shift 30 years ago with the realization that viral oncogenes have cellular counterparts. Since then, the identification and characterization of oncogenes that are activated by enhanced expression or mutation, and of tumor suppressor genes that are inactivated by reduced expression or mutation, has dominated research. This molecular revolution has included increasingly sophisticated gene expression analyses that have led to the concept of gene signatures. Such approaches reached a zenith with the application of next-generation sequencing to the characterization of cancer cell genomes and transcriptomes.

Most human cancers are solid tumors that arise from glandular epithelia; these adenocarcinomas account for the preponderance of cancer-associated morbidity and death worldwide. It is instructive to consider the impact of the above endeavour on the clinical management and outcome of patients with adenocarcinoma. There are examples, notably in colorectal, breast and lung cancer, of consequent introduction of effective treatments and useful biomarkers, however, for the vast majority of patients, treatment and survival have not been affected; improved outcome is explained by earlier diagnosis, reduced surgical morbidity, the introduction of adjuvant therapy and advances in systemic cytotoxic and endocrine therapies. Implementation of effective biomarkerdependent patient stratification should be a major goal of molecular medicine.

It is accepted that cancer is a genetic disease caused by stochastic accumulation of changes in gene sequence, regulation and expression. Notwithstanding, to take breast cancer as an example, the major gene signatures are defined, with the exception of HER2, by expression or absence of expression of genes that are expressed by normal breast epithelia. This observation illustrates that cancers are and should be defined not only by causative genetic and epigenetic events, but also by the extent to which they retain normal gene expression. Adenocarcinomas arise from glandular tissues and the malignant cells are characterized largely by their similarity to the progenitor cells. Appreciation of this phenotype provides insight into their cancer cell biology. Beatson made his breakthrough because he extrapolated from endocrine effects of the ovary on the normal mamma [1].

Trefoil proteins are expressed by all glandular, mucus-secretory epithelia. The questions are: is this normal expression retained by malignant cells and if it is, what are the consequences? Are trefoil proteins mutated in many human tumors? The answer is probably no. Are trefoil proteins important in human tumors? The answer is probably yes. If we understand how they are important then we can ask intelligent questions about their potential as biomarkers. The value of trefoil proteins as biomarkers in human neoplasias cannot be realized in the absence of an appreciation of their biology.

\section{Normal biology of trefoil proteins}

The human trefoil factor family comprises three small secreted proteins that are expressed almost exclusively by mucus-secretory epithelia $[2,3]$. Different epithelia are characterized by secretion of usually one and at most two members of the trefoil factor family. For instance, TFF1 is expressed by mucus-secretory cells that line the stomach, TFF2 by the more basal gastric epithelial

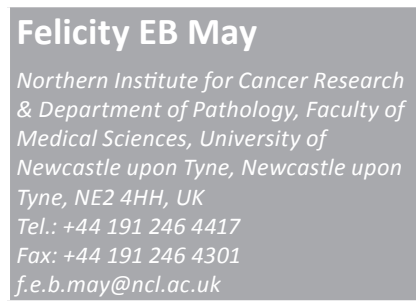

\section{Future $\mathrm{fSS}$}


cells and Brunner's glands of the duodenum, and TFF3 by the goblet cells of the intestines.

Trefoil proteins are defined by the presence of a 42-43-amino acid residue trefoil domain that forms a unique structural motif. Mature secreted TFF2 has 106 amino acid residues and contains two trefoil domains. Secreted TFF1 and TFF3 contain 60 and 59 amino acid residues, respectively, and have a single trefoil domain. A seventh cysteine residue outside the trefoil domain forms intermolecular disulfide bonds and facilitates the formation of TFF1 and TFF3 homodimers [4]. A TFF1 heterodimer of approximately $67 \mathrm{kDa}$ is secreted by breast cancer cells [5]. The major molecular form of TFF1 in gastric mucosa is a $25 \mathrm{kDa}$ heterodimer in which TFF1 is bound covalently to TFIZ1/GKN2, which is an $18.3 \mathrm{kDa}$ secreted protein of unknown function [6].

"The value of trefoil proteins as biomarkers in human neoplasias cannot be realized in the absence of an appreciation of their biology."

The normal function of trefoil proteins is thought to be maintenance of the integrity of and repair of mucosal surfaces. They achieve this by inhibition of cell growth and stimulation of cell motility and invasion, and have been implicated in mucus production, stability and function [7].

\section{Potential role in human cancer}

TFF1 was discovered as a consequence of its regulation by estrogen in breast cancer cells [8]. More recently, the induction of TFF3 by estrogen was recognized [9]. Estrogens drive breast cancer progression and their inhibition is clinically beneficial. An important question is, could this therapeutic benefit derive, in part, because trefoil protein expression and secretion is reduced? Many of the functions ascribed to trefoil proteins are suggestive of a promotional role in tumorigeneis. In vitro studies that indicate a role in the promotion of angiogenesis have been supported by translational work in gastric [10] and breast cancer [11]. Animal models of, for instance, pancreatic cancer, support a role in metastasis [12], and in breast cancer, TFF1 and TFF3 expression predicts bone metastasis [13]. In apparent contradiction, demonstration that TFF1-null mice develop early gastric cancers [7] and that approximately $50 \%$ of human gastric tumors do not express TFF1 [14], suggests that TFF1 might be a tumor suppressor.

Confusion remains in the field regarding the extent to which trefoil protein expression by malignant epithelial cells is ectopic. Initially, it was thought that TFF1 was not expressed by normal breast epithelia. Similarly, expression of TFF1 in pancreatic adenocarcinoma was wrongly considered to be ectopic. Misconceptions may have arisen due to insensitive assays or because expression may be regulated and hence variable; for instance, in breast epithelial cells during the menstrual cycle [11] and throughout a woman's reproductive life [15]. Malignancies that arise in metaplastic tissue cause confusion. TFF1 and TFF2 are not expressed in squamous epithelium but are expressed in Barrett's metaplasia of the esophagus. Expression of TFF1 and TFF2 in esophageal adenocarcinomas that arise in a background of Barrett's metaplasia should be regarded as retained rather than ectopic, and absence of expression should be regarded as loss of expression. The same argument applies to expression of TFF3 in intestinal-type gastric adenocarcinoma that arises in a background of intestinal metaplasia.

Trefoil factors are considered by some to be beneficial proteins whose loss militates towards cancer $[7,16]$, and by others as detrimental proteins whose gain promotes a more aggressive phenotype [17-19]. This divergence of opinion has divided the field. Recent work in the setting of breast cancer provides support for both camps and a rational resolution of this conundrum [11]. It is clear that TFF3 is expressed in and undergoes polarized secretion from normal lobular and ductal epithelial cells into the lumina. In younger women, the pattern of expression and secretion varies with the menstrual cycle. Retention of expression and reversed polarised secretion of TFF3 from invasive tumor cells is associated with muscle and skin infiltration, lymphovascular metastasis, involvement of lymph nodes and extracapsular spread. Thus it is not that TFF3 changes its function, it is that TFF3 functions in a different milieu.

\section{The future for trefoil proteins as biomarkers}

In light of the above, what is the future for trefoil proteins as biomarkers in human cancer as we move into the era of stratified medicine? Five main areas require consideration. The first is a consequence of trefoil factors being secreted proteins; their effect is felt beyond their cell of origin. Therein lies the crux of the question; which cells are being stimulated by the proteins? In normal breast epithelia and in well-differentiated areas of tumors, TFF3 is secreted into the lumina and exerts its normal protective influence on the epithelial mucosal layer. In invasive tumors or in areas of invasion, secretion 
is into the stroma, which posits TFF3 in the vicinity of adjacent malignant and stromal cells. In this situation, TFF3 may have a more malign influence, to drive tumor cells down the metastatic cascade by repression of cell growth and death and promotion of invasion both directly on the malignant cells and indirectly on the vasculature. The question is not only do the malignant cells express the proteins, but also to where are the proteins being secreted? Put another way, if trefoil proteins are being secreted by normal or quasinormal epithelial cells into ductal lumina, this is indicative of good differentiation and prognosis, but if they are being secreted by invasive malignant cells into the stroma then this is indicative of a metastatic phenotype. For example, expression and secretion of TFF1 from the gastric mucosa into the gastric lumen is normal and beneficial, but secretion of TFF1 by malignant gastric epithelial cells into the stroma is bad news.

The second consideration is whether different molecular forms of trefoil proteins should be discriminated. Available evidence suggests that they should. In vitro studies have shown that the TFF1 dimer has greater potency than the monomer [20]. Translational studies demonstrate that gastric tumors that express TFF1 are more likely to metastasize if the heterodimer is absent [21].

\section{"Trefoil proteins may have value as predictive biomarkers both of responsiveness and of the potential worth of endocrine therapy."}

The third area is whether or not TFF1 and TFF3 are predictive markers of response to endocrine therapy. There is substantive evidence in support of TFF1 $[22,23]$. The jury has not been but should be convened for TFF3. Likewise, their ability to predict response to hormonal therapy should be investigated in other tumor types [24].

The fourth aspect is the relationship of trefoil proteins to metastasis. TFF3 discriminates between more or less metastatic cells; higher expression was associated with propensity to invade the lymphovasculature or the axilla. Notably, TFF3 expression discriminated grade 3 breast tumors [11]. Consideration should be given to the possibility that trefoil proteins are prognostic biomarkers whose measurement could supplement nodal status and identify low-histological grade tumors with worse prognosis or high-histological grade tumors with better prognosis [11].

The fifth area is how intratumor heterogeneity affects the value of trefoil proteins as biomarkers. Presumably for prognostic biomarkers of invasion and metastasis, expression by even a minority of cells will be of import. For predictive biomarkers, it is critical to decipher the proportion of cells that will be targeted successfully. A patient might achieve little long-term benefit from endocrine therapy if only $10 \%$ of the tumor cells respond. However, the effect of secreted proteins is felt a priori beyond the originator cell. If those $10 \%$ of responsive tumor cells express and secrete proteins known to promote invasive phenotypes, then targeted elimination of those tumor cells may be clinically significant. Trefoil proteins may have value as predictive biomarkers both of responsiveness and of the potential worth of endocrine therapy.

In conclusion, the majority of biomarker studies are predicated on simple linear associations between total RNA or protein levels and clinical parameters. Trefoil proteins are a textbook example of how a nuanced approach in which analysis of expression of different molecular forms of the protein in the context of tumor architecture is required.

\section{Financial \& competing interests disclosure}

The author has no relevant affliations or financial involvement with any organization or entity with a financial interest in or financial conflict with the subject matter or materials discussed in the manuscript. This includes employment, consultancies, honoraria, stock ownership or options, expert testimony, grants or patents received or pending, or royalties.

No writing assistance was utilized in the production of this manuscript.

\section{References}

1 Beatson GT. On the treatment of inoperable cases of carcinoma of the mamma:

suggestions for a new method of treatment, with illustrative cases. Lancet 11148 (3802), 104-107 (1896).

2 May FEB, Westley BR. Trefoil proteins: their role in normal and malignant cells. J. Pathol. 183(1), 4-7 (1997).
Thim L, May FEB. Structure of mammalian trefoil factors and functional insights. Cell. Mol. Life Sci. 62(24), 2956-2973 (2005).

4 May FEB, Church ST, Major S, Westley BR. The closely related estrogen-regulated trefoil proteins TFF1 and TFF3 have markedly different hydrodynamic properties, overall charge, and distribution of surface charge. Biochemistry 42(27), 8250-8259 (2003).
5 Chadwick MP, Westley BR, May FEB. Homodimerization and heterooligomerization of the single-domain trefoil protein pNR-2/pS2 through cysteine Biochem. J. 327(Pt 1), 117-123 (1997).

6 Westley BR, Griffin SM, May FEB. Interaction between TFF1, a gastric tumor suppressor trefoil protein, and TFIZ1, a brichos domaincontaining protein with homology to SP-C. Biochemistry 44(22), 7967-7975 (2005). 
7 Lefebvre O, Chenard MP, Masson R et al. Gastric mucosa abnormalities and tumorigenesis in mice lacking the $\mathrm{pS} 2$ trefoil protein. Science 274(5285), 259-262 (1996).

- Provided the first evidence that TFF1 might be a tumor suppressor. The authors demonstrated that some TFF1-null mice develop early gastric carcinomas.

8 Masiakowski P, Breathnach R, Bloch J, Gannon F, Krust A, Chambon P. Cloning of cDNA sequences of hormone-regulated genes from the MCF-7 human breast cancer cell line. Nucleic Acids Res. 10(24), 7895-7903 (1982).

9 May FEB, Westley BR. Expression of human intestinal trefoil factor in malignant cells and its regulation by oestrogen in breast cancer cells. J. Pathol. 182(4), 404-413. (1997).

10 Dhar DK, Wang TC, Tabara $\mathrm{H}$ et al. Expression of trefoil factor family members correlates with patient prognosis and neoangiogenesis. Clin. Cancer Res. 11(18), 6472-6478 (2005).

- Provided the first translational evidence for the role of trefoil proteins in the promotion of tumor angiogenesis.

11 Ahmed AR, Griffiths AB, Tilby MJ, Westley $\mathrm{BR}$, May FEB. TFF3 is a normal breast epithelial protein and is associated with differentiated phenotype in early breast cancer but predisposes to invasion and metastasis in advanced disease. Am. J. Pathol. 180(3), 904-916 (2012).

- This first study to measure TFF3 protein in breast cancer tissue demonstrated that its expression is associated both with features of good prognosis and with metastases, which confirmed the paradox that trefoil factors are associated with good and bad clinicopathological parameters.
12 Arumugam T, Brandt W, Ramachandran V et al. Trefoil factor 1 stimulates both pancreatic cancer and stellate cells and increases metastasis. Pancreas 40(6), 815-822 (2011).

13 Smid M, Wang Y, Klijn JGM et al. Genes associated with breast cancer metastatic to bone. J. Clin. Oncol. 24(15), 2261-2267 (2006).

- This comprehensive study measured gene expression with expression arrays in early lymph node-negative breast cancers. Expression was correlated with subsequent development of bone metastases. Of the 31 genes capable to predict bone metastasis, TFF1 and TFF3 were the first and second most powerful predictors, respectively.

14 Henry JA, Bennett MK, Piggott NH, Levett DL, May FEB, Westley BR. Expression of the $\mathrm{pNR}-2 / \mathrm{pS} 2$ protein in diverse human epithelial tumors. Br. J. Cancer. 64(4), 677-682 (1991).

15 Lee O, Helenowski IB, Chatterton RT Jr, Jovanovic B, Khan SA. Prediction of menopausal status from estrogen-related gene expression in benign breast tissue. Breast Cancer Res. Treat. 131(3), 1067-1076. (2012).

16 Buache E, Etique N, Alpy F et al. Deficiency in trefoil factor 1 (TFF1) increases tumorigenicity of human breast cancer cells and mammary tumor development in TFF1knockout mice. Oncogene 30(29), 3261-3273 (2011).

17 Yamachika T, Werther JL, Bodian C et al. Intestinal trefoil factor: a marker of poor prognosis in gastric carcinoma. Clin. Cancer Res. 8(5), 1092-1099 (2002).

Provided strong support for the contention that trefoil factors are markers of poor prognosis. The authors demonstrated that patients whose gastric adenocarcinomas expressed TFF3 had shorter overall survival.
18 Mikhitarian K, Gillanders WE, Almeida JS et al. An innovative microarray strategy identities informative molecular markers for the detection of micrometastatic breast cancer. Clin. Cancer Res. 11(10), 3697-3704 (2005).

19 Crosier M, Scott D, Wilson RG, Griffith CDM, May FEB, Westley BR. High expression of the trefoil protein TFF1 in interval breast cancers. Am. J. Pathol. 159(1), 215-221 (2001).

20 Prest SJ, May FEB, Westley BR. The estrogen-regulated protein, TFF1, stimulates migration of human breast cancer cells. FASEB J. 16(6), 592-594 (2002).

21 May FEB, Griffin SM, Westley BR. The trefoil factor interacting protein TFIZ1 binds the trefoil protein TFF1 preferentially in normal gastric mucosal cells but the co-expression of these proteins is deregulated in gastric cancer. Int. J. Biochem. Cell Biol. 41(3), 632-640 (2009).

- This is the first demonstration that a member of the trefoil factor family of proteins is bound covalently to a brichos domain-containing protein.

22 Henry JA, Piggott NH, Mallick UK et al. pNR-2/pS2 immunohistochemical staining in breast cancer: correlation with prognostic factors and endocrine response. Br. J. Cancer. 63(4), 615-622 (1991).

23 Foekens JA, Portengen H, Look MP et al. Relationship of PS2 with response to tamoxifen therapy in patients with recurrent breast cancer. Br. J. Cancer. 70(6), 1217-1223 (1994).

24 Rickman DS, Chen YB, Banerjee S et al. ERG cooperates with androgen receptor in regulating trefoil factor 3 in prostate cancer disease progression. Neoplasia 12(12), 1031-1040 (2010). 